\title{
Unilateral nevoid hyperkeratosis of the nipple and areola in a Saudi female
}

\section{Taghreed Mahjoub}

\author{
King Abdullah Medical Complex- Jeddah, Saudi Arabia \\ Corresponding author: Dr. Taghreed Mahjoub, E-mail: dr.taghred@hotmail.com
}

\begin{abstract}
Nevoid hyperkeratosis of the nipple and areola (NHNA) is a rare, benign condition of unknown origin that is characterized by a hyperpigmented, hyperkeratotic verruca plaque over the nipple and/or areola. NHNA usually occurs bilaterally, but is sometimes present unilaterally. We present a case of a 21 -year-old Saudi female who presented at dermatology outpatient clinic with a 3-year history of skin darkening and thickening of the left nipple, associated with moderate itching. The patient was diagnosed with unilateral NHNA on the basis of clinical presentation and skin-punch biopsy findings of epidermal orthokeratotic hyperkeratosis, mild papillomatosis, basal-layer hyperpigmentation and irregular filiform-pattern epidermal acanthosis. To the best of our knowledge, this case represents the first report of NHNA with unilateral presentation in a Saudi female.
\end{abstract}

key words: Nevoid hyperkeratosis; Nipple; Areola

\section{INTRODUCTION}

Nevoid hyperkeratosis of the nipple and areola (NHNA) was first described by Taber in 1932 [1]. Hyperkeratosis of the areola and nipple was classified by Levy-Franckel into three types: type 1 is characterized by an extension of the epidermal nevus; type 2 occurs secondary to another form of dermatosis; and type 3 is the idiopathic, isolated form [2]. However, Pérez-lzquierdo et al. suggested an alternative classification that distinguished just two types of nipple hyperkeratosis: either idiopathic (nevoid) or occurring secondary to other cutaneous conditions [3].

Nevoid hyperkeratosis of the nipple and areola is characterized by a hyperpigmented, hyperkeratotic verruca plaque that involves the nipple and/or areola $[3,4]$. This condition is rare, and most frequently occurs bilaterally, although unilateral cases have also been reported [5-7].

NHNA is a clinical entity that tends to be diagnosed on the basis of the exclusion of other conditions. The patient who presents with hyperkeratosis of the nipple and/or areola must be examined carefully for the presence of other underlying cutaneous diseases, including epidermal nevi, ichthyosis, acanthosis nigricans, Darier disease, and cutaneous T-cell lymphoma. If no other diagnosis is suggested by the clinical findings, then a diagnosis of NHNA can be made.

Here, we report a case of unilateral NHNA in a 21-yearold female from Saudi Arabia. The patient presented with a left-nipple hyperpigmented, hyperkeratotic verruca plaque with a histopathological finding of orthokeratotic hyperkeratosis and papillomatosis, with mild epidermal acanthosis. Diagnosis was made on the basis of clinical presentation and histopathological findings, as well as the exclusion of other diagnoses.

\section{CASE REPORT}

An otherwise healthy, 21-year-old, single Saudi female presented to the outpatient dermatology clinic with a 3-year history of progressive skin thickening and darkening of the left nipple, associated with moderate

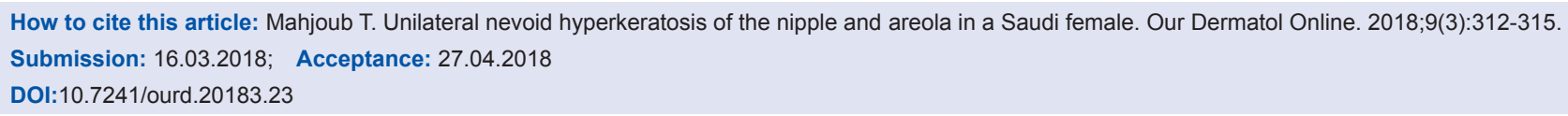


itching. The patient had no history of nipple discharge or bleeding, breast pain or masses, skin lesions at other sites of the body, drug use, personal or family history of atopy or family history of a similar skin condition.

On physical examination, asymmetry was observed between the patient's breasts, with normal appearance of the right breast, but a diffuse, brown, hyperpigmented, hyperkeratotic verruca plaque over the left nipple and areola (Figs. 1, 2A and 2B). Bilateral examination of the breasts found no mass, tenderness or discharge. There was no evidence of lymphadenopathy. An examination of the skin of the neck, axillae and other intertriginous areas showed no increase in pigmentation or thickening. Similarly, the nails, hair and skin covering other areas of the body were normal. A 4-mm skin-punch biopsy was performed. Examination of the skin punch biopsy indicated the presence of epidermal orthokeratotic hyperkeratosis, mild papillomatosis, basal-layer hyperpigmentation and an irregular pattern of epidermal acanthosis with elongated rete ridges (Figs. 3 and 4).

The diagnosis of unilateral NHNA was made on the basis of the clinical and the histopathological findings. In addition, secondary causes of nipple hyperkeratosis, such as acanthosis nigricans, seborrheic keratosis, mammary Paget's disease, atopic dermatitis, superficial basalcell carcinoma and mycosis fungoides, were excluded. Using the Levy-Franckel classification, our patient was classified as having type III (i.e., idiopathic) NHNA.

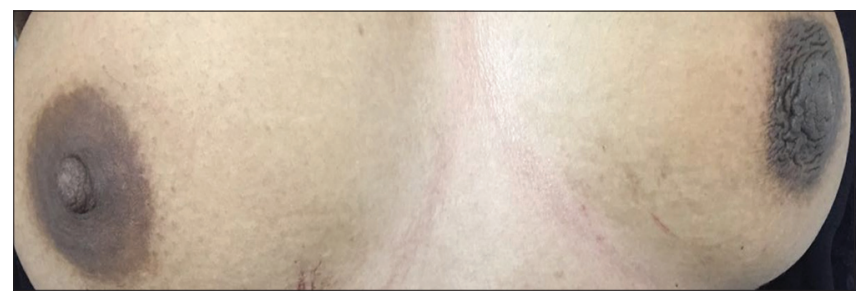

Figure 1: Photograph of the patient, showing unilateral nevoid hyperkeratosis of the nipple and areola on the left breast, compared with the normal tissue of the right breast.

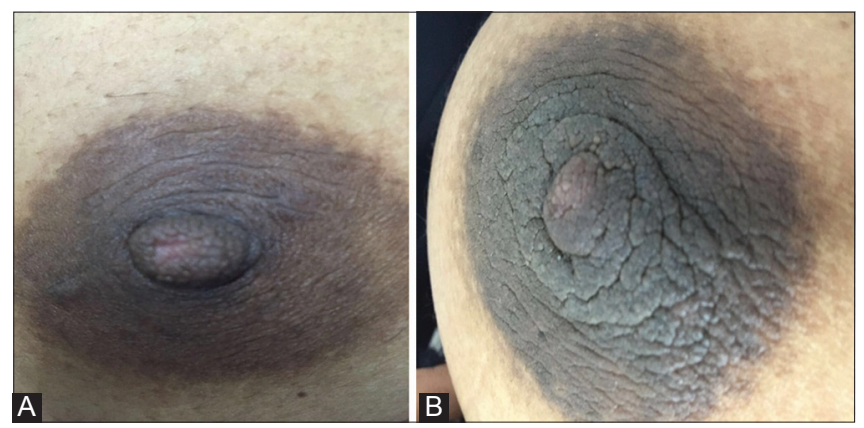

Figure 2: A magnified view showing $(A)$ the normal right breast and (B) the verrucous, hyperpigmented thickening of the left areola and nipple.
The patient was treated with calcipotriol (synthetic vitamin D3) cream daily for 6 months, at the end of which she was satisfied with the cosmetic result, reporting the disappearance of itching and a 50\% decrease in skin thickening of the left nipple and areola. There was no treatment-related skin irritation.

Prior to the study, patient gave written consent to the examination and biopsy after having been informed about the procedure.

\section{DISCUSSION}

The present case report identified a Saudi female with idiopathic hyperkeratosis of the nipple and areola. Notably, $80 \%$ of 45 cases identified in a Review of NHNA had occurred in female patients [8].

Although the exact cause of NHNA remains unknown, it has been postulated that some cases could arise from

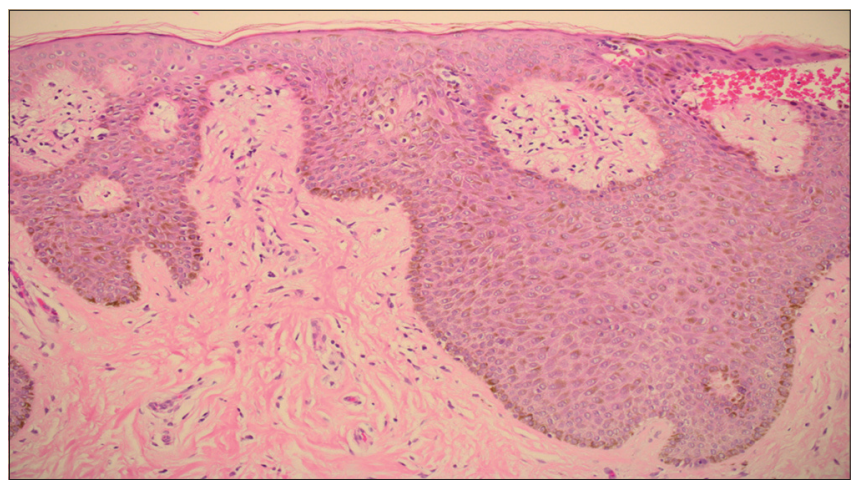

Figure 3: Skin biopsy from the patient's left breast, stained with hematoxylin and eosin and, 400x original magnification, showing orthokeratotic hyperkeratosis, basal-layer hyperpigmentation and irregular pattern acanthosis with elongated rete ridges.

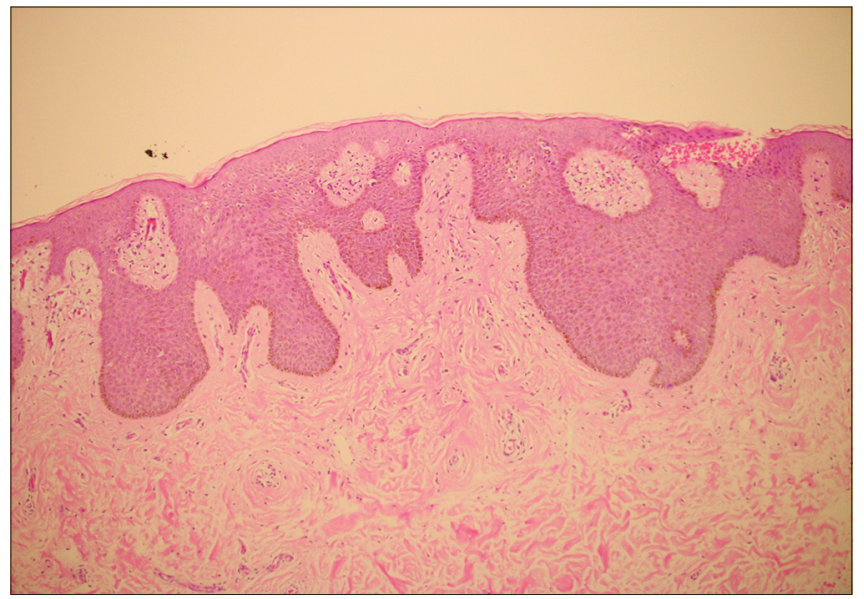

Figure 4: A skin biopsy from the patient's left breast, stained with hematoxylin and eosin and presented at $100 x$ original magnification, to show irregular filiform epidermal acanthosis with elongated rete ridges. 
an alteration in estrogen levels [9]. In support of this hypothesis, two cases of NHNA have been reported in an elderly male who received estrogen therapy for prostatic adenocarcinoma [8]. Another patient developed NHNA during pregnancy [10]. In a study of female patients with NHNA, six of the seven cases examined had occurred among women of reproductive age, who were in the second or third decade of life [3]. Similarly, our patient had started to develop symptoms 3 years before she first presented to the dermatology clinic at 21 years old.

More than half of the cases of NHNA occur bilaterally, but unilateral cases have been reported [5-7]. Our patient presented with the less common unilateral type of NHNA.

Histopathological findings of NHNA include epidermal orthokeratotic hyperkeratosis, papillomatosis and occasional keratin plugging with irregular filiformpattern epidermal acanthosis. The basal layer of epidermis is hyperpigmented, without melanocyte proliferation, and the dermis may show mild perivascular lymphocytic infiltration $[3,4,6,7,11]$. Our case was diagnosed clinically and confirmed with a left-nipple histopathological finding of epidermal orthokeratotic hyperkeratosis, mild papillomatosis, basal-layer hyperpigmentation and an irregular pattern of epidermal acanthosis with elongated rete ridges.

Different therapeutic options have been used to manage NHNA, with varying results. The use of topical calcipotriol (a synthetic form of vitamin D3) has been reported to be effective and to produce a rapid outcome [5]. Topical calcipotriol combined with topical tacrolimus (an immunosuppressant) has previously been used to treat NHNA in a 19-year-old female, with improvement noted after 2 months of treatment [12]. Low-dose oral acitretin (a secondgeneration retinoid) has also been combined with topical calcipotriol for the treatment of NHNA, with no relapse after 2 years of follow-up [13].

The mechanism of action of topical calcipotriol in NHNA could involve inhibition of cellular proliferation and induction of keratinocyte differentiation. Topical calcipotriol is considered a very safe drug when used in amounts up to $100 \mathrm{~g}$ per week. The most important adverse effect of topical calcipotriol is skin irritation, but it also has a potential effect on calcium hemostasis [14]. Our patient was treated with topical calcipotriol cream daily for 6 months, and she experienced disappearance of skin itching, and a $50 \%$ decrease in skin thickening of the left nipple.

Acceptable cosmetic results have been obtained after the treatment of NHNA with a topical retinoid [15]. Cryotherapy has also been used successfully in the treatment of NHNA, with improvement noted after five sessions (with $20 \mathrm{~s}$ of cryotherapy per session) in a female patient who failed to respond to topical keratolytic agents [16]. In addition, surgical treatment modalities have been described, including radiofrequency surgery [17].

To the best of our knowledge, this case provides the first report of unilateral NHNA in Saudi Arabia. Although this condition is benign, it is considered distressing for the patient because of a disfigured appearance and of concern to doctors because of its similarity to mammary Paget's disease.

\section{ACKNOWLEDGEMENTS}

I would like to thank Dr. Awadh Al Amri and Dr.Louai Salah for reviewing the manuscript. Also I would like to thank King Abdullah International Medical research center for the article English language editing.

\section{CONSENT}

The examination of the patient was conducted according to the Declaration of Helsinki principles.

\section{REFERENCES}

1. Oberste-Lehn H. Hyperkeratosis in the region of the nipple and areola. Z Haut Geschlechtskr 1950;8:388-93.

2. Levy-Franckel A. Les hyperkeratosis de I'areola et du mamelon $\mathrm{r}$. Paris Med. 1938;28:63-6.

3. Baykal C, Büyükbabani N, Kavak A, Alper M. Nevoid hyperkeratosis of the nipple and areola: a distinct entity. J Am Acad Dermatol. 2002;46:414-8.

4. Aytekin S, Tarlan N, Alp S, Uzunlar A. Naevoid hyperkeratosis of the nipple and areola. J Eur Acad Dermatology Venereol. 2003;17:232-3.

5. Raffas W, El Amrani F, Benzekri L, Benzekri A, Senouci K, Hassam B. Successful Treatment with Calcipotriol for Nevoid Hyperkeratosis of the Nipples. Clin Med Insights Dermatol. 2013;6:27-30.

6. D'Souza M, Gharami R, Ratnakar C, Garg BR. Unilateral nevoid hyperkeratosis of the nipple and areola. Int J Dermatol. 1996;35:602-3.

7. Hariharasubramony A, Chankramath S. Hyperkeratosis of nipple and areola. Our Dermatol Online. 2012;3:215-6.

8. Obayashi H, Tsuchida T, Ikeda S. Hyperkeratosis of the nipple and areola. Rinsho Dermatol. 1998;147-50. 
9. Mold DE, Jegasothy B V. Estrogen-induced hyperkeratosis of the nipple. Cutis. 1980;26:95-6.

10. Higgins HW, Jenkins J, Horn TD, Kroumpouzos G. PregnancyAssociated Hyperkeratosis of the Nipple. JAMA Dermatol. 2013;149:722.

11. Yazdabadi A, Green J, Sinclair R. Successful treatment of femalepattern hair loss with spironolactone in a 9-year-old girl. Australas J Dermatol. 2009;50:113-4.

12. Tran VT, Nguyen T Van, Hoang M Van. Case report: Nevoid hyperkeratosis of the nipple and areola : Treatment with topical calcipotriol and topical tacrolimus. J Am Acad Dermatol. 2016;74:AB47.

13. Kartal Durmazlar SP, Eskioglu F, Bodur Z. Hyperkeratosis of the nipple and areola: 2 years of remission with low dose acitretin and topical calcipotriol therapy. J Dermatolog Treat. 2008;19:337-40.

14. Fogh K, Kragballe K. Vitamin D3 analouges. Dermatol Ther.
1999;11:20-31.

15. Pérez-Izquierdo JM, Vilata JJ, Sánchez JL, Gargallo E, Millan F, Aliaga A. Retinoic acid treatment of nipple hyperkeratosis. Arch Dermatol. 1990;126:687-8.

16. Vestey JP, Bunney MH. Unilateral Hyperkeratosis of the Nipple: The response to cryotherapy. Arch Dermatol. 1986;122:1360.

17. Ozyazgan I, Kontaş O, Ferahbaş A. Treatment of nevoid hyperkeratosis of the nipple and areola using a radiofrequency surgical unit. Dermatol Surg. 2005;31:703-5.

Copyright by Taghreed Mahjoub. This is an open-access article distributed under the terms of the Creative Commons Attribution License, which permits unrestricted use, distribution, and reproduction in any medium, provided the original author and source are credited.

Source of Support: Nil, Conflict of Interest: None declared. 